\title{
Coherent Doppler narrowing in a thin vapor cell: Observation of the Dicke regime in the optical domain
}

\author{
Stephan Briaudeau, ${ }^{1}$ Solomon Saltiel, ${ }^{1, *}$ Gerard Nienhuis, ${ }^{2}$ Daniel Bloch,,${ }^{1, \dagger}$ and Martial Ducloy ${ }^{1}$ \\ ${ }^{1}$ Laboratoire de Physique des Lasers, UMR 7538 du CNRS, Institut Galilée, \\ Université Paris-Nord, F-93430 Villetaneuse, France \\ ${ }^{2}$ Huygens Laboratory, Rijksuniversiteit, Leiden, Postbus 9504, 2300 RA Leiden, The Netherlands
}

(Received 12 January 1998)

\begin{abstract}
Single light beam transmission spectroscopy in an ultrathin dilute vapor cell $(10-100 \mu \mathrm{m})$ reveals subDoppler features. Indeed, atoms with fast (normal) velocity undergo a short interaction between the cell walls, and are shown to be less absorbing because they have not reached their steady state of interaction with light. The effect is observed in the linear-response regime with a simple two-level system, as ensured from the ultralow level of light irradiation $\left(\leqslant 1 \mu \mathrm{W} / \mathrm{cm}^{2}\right.$ for the Cs $D_{2}$ line). The effect, known for a long time in the microwave domain, is demonstrated here in the optical domain, and is extended to cell thicknesses large relative to the optical wavelength. [S1050-2947(98)50105-9]

PACS number(s): 42.50.Md, 32.80.-t, 06.20.-f
\end{abstract}

High-resolution spectroscopy of an atomic vapor with a single-frequency laser beam is often considered as the firm basis on which numerous experiments daily rely. In spite of the apparent simplicity of these types of experiments, recent careful observations have demonstrated that various elementary effects, which are often neglected, may appear in commonly encountered regimes. Recently, we demonstrated that a strong anisotropy in the design of an otherwise ordinary room-temperature vapor cell leads to sub-Doppler features on line center in the spectrum of single beam transmission [1]. This observation was shown to be related to an optical pumping effect, whose steady state is reached only for those atoms whose vectorial velocity ensures a long interaction time with the resonant pumping light, i.e., atoms with long free flights between the cell walls. Here, we demonstrate a simple effect, occurring in a similar thin vapor film, under conditions of extremely weak light irradiation. Indeed, the sub-Doppler contribution that is reported here simply originates in the transient regime of the linear interaction with a two-level system. As shown below, this elementary effect generalizes, into the optical domain, an effect first discussed by Dicke and co-worker [2] in the context of microwave spectroscopy, for an rf cavity with a "pillbox geometry" and a thickness $\sim \lambda_{\text {rf }}$.

Our experimental setup is essentially similar to the one used previously [1] in which a weak laser beam, issued from a tunable, frequency-modulated diode laser, is sent under normal incidence to a (commercially available) thin glass cell (thickness in the range 10-100 $\mu \mathrm{m}$ ), filled with lowpressure vapor. The laser-beam transmission is monitored with an ultrasensitive photodiode (maximum light power is $50 \mathrm{nW}$ with our currently used detector, Hamamatsu C-5460$01-)$, and the signal is recorded through a phase-sensitive detection, synchronous to the applied FM, hence yielding the

\footnotetext{
*Also at University of Sofia, Bulgaria.

${ }^{\dagger}$ Author to whom correspondence should be addressed. FAX: 331494032 00. Electronic address: bloch@1pl.univ-paris13.fr
}

frequency derivative of the transmission spectrum. Typical spectra obtained on the Cs $D_{2}$ line $(852 \mathrm{~nm})$ are presented in Fig. 1. One notes narrow Doppler-free structures, superimposed to the dispersionlike background, associated with the frequency derivative of the Doppler-broadened transmission. As deduced from a comparison with this Doppler-broadened background, these narrow resonances, which are centered on the resonance frequency of the various hyperfine components, correspond to a relative increase of absorption at line center relatively to the absorption in the wings. Note that in most experimental situations these small sub-Doppler contri-

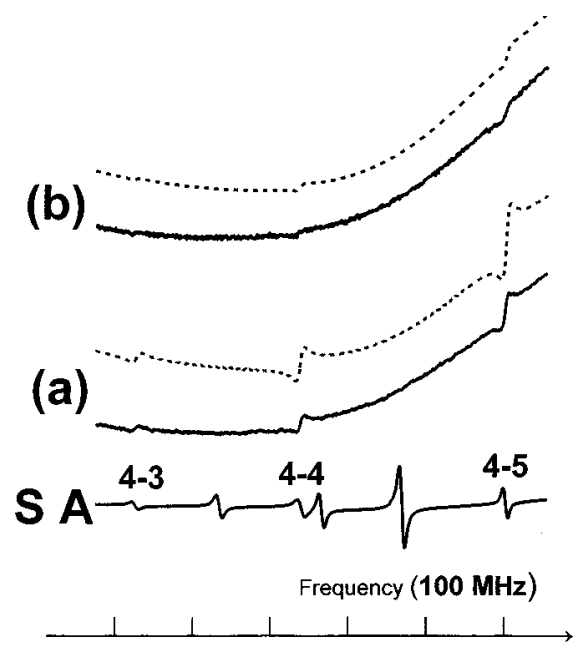

FIG. 1. Transmission spectrum through a thin Cs cell, as observed after demodulation of the applied FM. Full line spectra, experimental data with cell thickness (a) $10 \mu \mathrm{m}$ or (b) $50 \mu \mathrm{m}$; the incident power is $\sim 50 \mathrm{nW}$, the beam diameter is $\sim 4 \mathrm{~mm}$, and the various narrow lines are the hyperfine components of the 852-nm line $6 S_{1 / 2}(F=4)-6 P_{3 / 2}\{F=3,4,5\}$. The simultaneously recorded saturated absorption (SA) spectrum is a frequency reference. The dashed lines above experimental data (a) and (b) are calculated spectra, assuming that $\gamma / k u_{\text {th }}=50$, with $L / \lambda=10$ and $L / \lambda=50$, respectively (for a Cs linewidth of $5 \mathrm{MHz}$ and a Doppler width of $\sim 250 \mathrm{MHz}$ ). Each unit of the horizontal frequency scale stands for $100 \mathrm{MHz}$. 
butions only become observable thanks to the FM technique that enhances the contrast of the narrower resonances. Assignment of these narrow resonances to an absorption increase is confirmed through a prior optical measurement of the phase of the applied FM, which also shows the short response time of these sub-Doppler contributions (in the $\mathrm{MHz}^{-1}$ range).

These observations were performed on several Cs cells (nominal thickness ranging from $10 \mu \mathrm{m}$ to $100 \mu \mathrm{m}$ ) that were heated up to temperatures for which the Dopplerbroadened absorption is $\sim 3-30 \%$ (i.e., $\sim 80-100{ }^{\circ} \mathrm{C}$ for a $10-\mu \mathrm{m}$ cell). A striking point is that the observed absorption behavior is independent of the incident light intensity in our range of operation (our ultraweak irradiation lies in the 1100-nW domain, for a 4-mm beam diameter). In particular, for a given cell length, the ratio between sub-Doppler contribution and the Doppler background is independent of the experimental conditions and, as shown in Fig. 1, calculated spectra, as derived from the model detailed below, fairly reproduce experimental observations. All these features establish that the presently observed sub-Doppler contribution is typical of the regime of linear absorption, in which the incident intensity is kept below the onset of both optical pumping and saturation. These results clearly differ from our previous observations [1] that were interpreted through a threelevel system in which velocity-selective optical pumping to a noninteracting level took place. The recovering of this optical pumping mechanism actually occurs for much larger intensity (the onset is on the order of $\sim 0.5 \mu \mathrm{W}$, depending upon the frequency of the FM); the narrow resonance then decreases with increasing power, and finally becomes inverted [3].

The theoretical frame for understanding the observed behavior, has been derived by several authors [4,5]: light absorption results from the destructive interference between the incident field and the summing up of the fields re-radiated by the optical dipoles that are induced by the excitation. Because of phase-interrupting collisional processes, the amplitude of these resonant dipoles is governed by their transient response to the incident optical excitation [6]. Under the following assumptions: (i) the vapor is dilute enough, so that only atom-surface collisions are to be considered, (ii) atoms

(a)

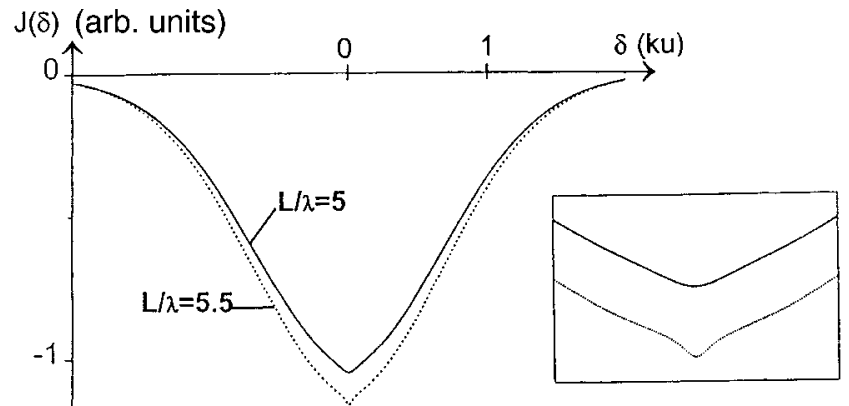

$J(\delta)$

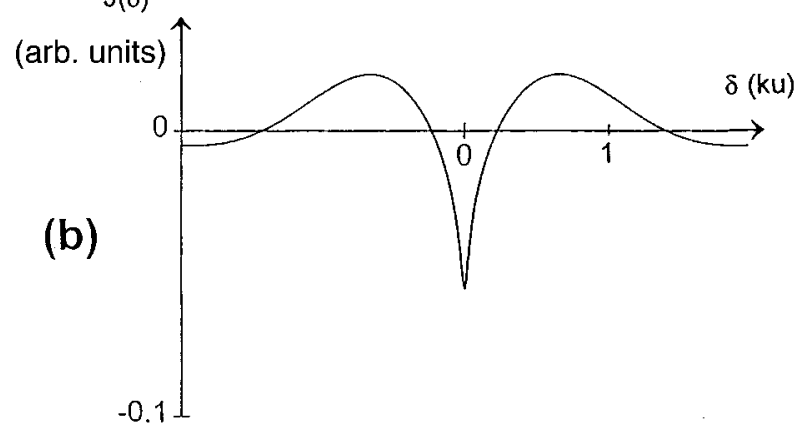

(d)

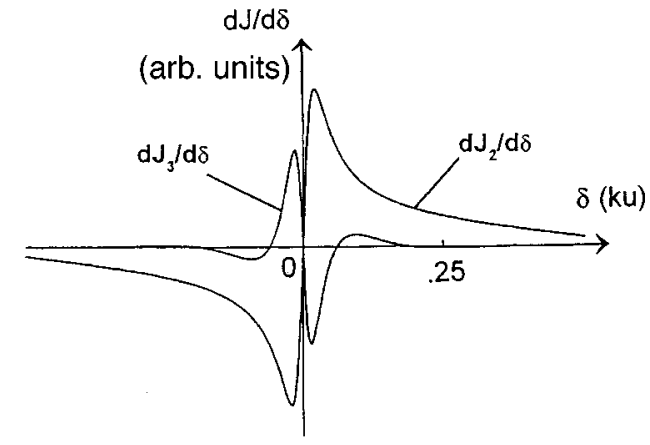

$\mathrm{J}(\delta)$
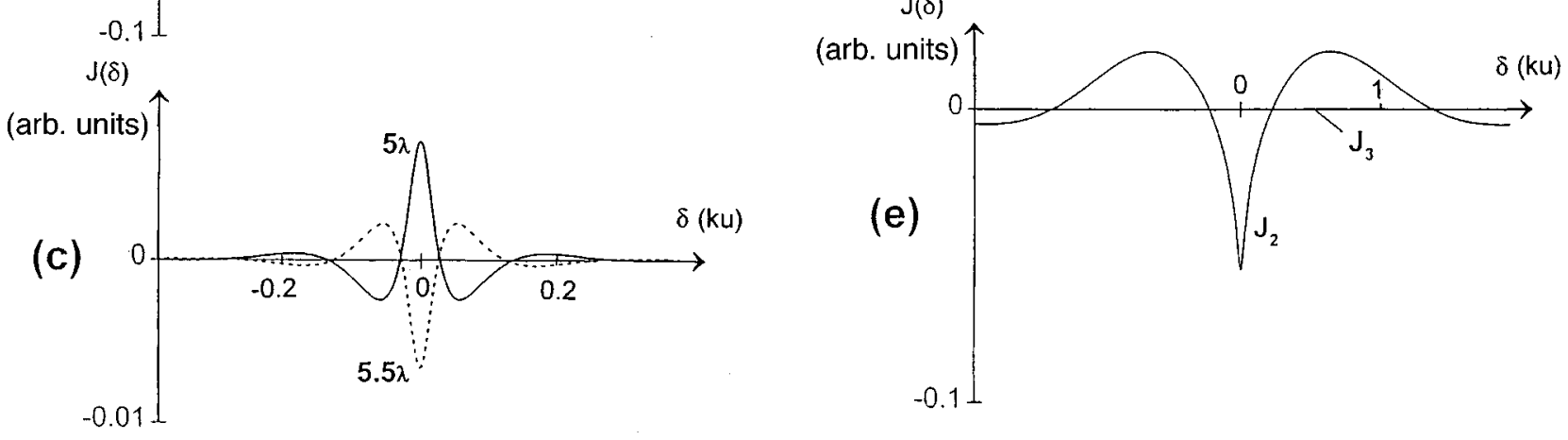

FIG. 2. Line shapes $J(\delta)$ or $d J / d \delta$ associated with the different terms $\operatorname{Re}\left[J_{1}\right], \operatorname{Re}\left[J_{2}\right]$, and $\operatorname{Re}\left[J_{3}\right]$, as calculated in the case $\gamma / k u_{\text {th }}$ $=50$; the vertical (arbitrary) unit is the same for (a), (b), (c), and (e). (a) Overall absorption line shape $J_{1}+J_{2}+J_{3}$ for $L=5 \lambda$, and for $L=5.5 \lambda$. The (interferometric) difference between 5 and $5.5 \lambda$ is more visible at line center (see the zoom at $\times 4$ ); (b) $J_{2}$, with the same vertical unit as in (a), calculated for $L=5 \lambda$ (note that $J_{2}$ is actually independent of $L$ ); (c) $J_{3}$ for $L=5 \lambda$, and for $L=5.5 \lambda$ [the same vertical unit as in (a); note the change in the frequency scale]; (d) $d J_{2} / d \delta$ and $d J_{3} / d \delta$ for $L=5 \lambda$. Note that for $L$ averaged between $4.5 \lambda$ and $5.5 \lambda$, $d\left(J_{2}+J_{3}\right) / d \delta$ overlaps $d J_{2} / d \delta$, with no visible difference at the present scale; (e) for $L=50 \lambda, J_{3}$ has turned out to be negligible relative to $J_{2}$. 
leave the wall in the ground state, (iii) the incident laser beam diameter largely exceeds the cell thickness, one derives the following line shape from a spatial integration of the transient atomic response

$$
\alpha(\omega) \propto \operatorname{Re} \int_{-\infty}^{+\infty} d v W(v) g\left(\omega-\omega_{0}, v, L\right),
$$

with

$$
g\left(\omega-\omega_{0}, v, L\right)=-\frac{k}{\Lambda}\left[L-\frac{|v|}{\Lambda}(1-\exp -\Lambda L /|v|)\right],
$$

and

$$
\Lambda=\gamma-i\left[\left(\omega-\omega_{0}\right)-k v\right]
$$

In Eqs. (1)-(3), $\alpha(\omega)$ is the absorption coefficient at the incident laser frequency $\omega, W(v)$ stands for the normalized distribution of the normal velocity component, $\omega_{0}$ and $\gamma$ respectively define the two-level atom transition frequency and optical width, and $k$ is the wave-vector modulus. For convenience, and following the respective terms appearing in $g\left(\omega-\omega_{0}, v, L\right)$, we also define the integrals $J_{1}, J_{2}, J_{3}$ :

$$
\begin{gathered}
J_{1}=-\int d v W(v) \frac{k L}{\Lambda}, \\
J_{2}=\int d v W(v) \frac{k|v|}{\Lambda^{2}}, \\
J_{3}=-\int d v W(v) \frac{k|v|}{\Lambda^{2}} \exp (-L \Lambda /|v|) .
\end{gathered}
$$

The $J_{1}$ term provides the usual steady-state Dopplerbroadened absorption expected in a macroscopic cell. The sub-Doppler features are hence associated with the transient effects as considered in the $J_{2}$ and $J_{3}$ terms. Indeed, the atomic free path along which the transient effects are evaluated, is actually highly anisotropic, and atoms flying nearly parallel to the wall yield a stronger contribution to the signal while their resonance appears unsensitive to the Doppler shift. It can be seen that $J_{2}$ originates in the buildup of the atomic response to the driving field, while $J_{3}$ reflects the finiteness of the interaction time $L / v$, which is limited by the cell thickness. Since $\Lambda$ is generally complex, $J_{3}$ includes an interferometric dependence upon the cell length, with a quasiperiod equal to the wavelength (see Fig. 2). In particular, the overall absorption at line center gets stronger when $L / \lambda$ is a half-integer [7], as can be seen from Refs. [4, 5]. This apparently severe length dependence is typical of a linear coherent transient effect, as opposed to the incoherent optical pumping mechanism in a thin cell as analyzed in Ref. [1].
However, it is worth noting that in the experimental cases of relatively large values of $L$ destructive interferences between the various velocity contributions tend to wash out the $\operatorname{Re}\left(J_{3}\right)$ term, making the $J_{2}$ contribution dominant (see Fig. 2 and Ref. [8]). This $\operatorname{Re}\left(J_{2}\right)$ contribution actually mixes up absorptive contribution [i.e., $\operatorname{Re}\left(\Lambda^{-1}\right)$, with tails in $v^{-2}$, so that a single velocity group provides a dominant contribution] and dispersive contribution [i.e., $\operatorname{Im}\left(\Lambda^{-1}\right)$, which spans as $\left.v^{-1}\right]$. A signature of the contribution of these tails of the velocity distribution can be seen in the fact that this $J_{2}$ term becomes negligible relative to $J_{1}$ when $L \gg u_{\text {th }} / \gamma\left(u_{\text {th }}\right.$ the most probable thermal velocity). Indeed, in this case, even the faster atoms $\left(v \approx u_{\text {th }}\right)$ are close to the steady-state regime of interaction with the optical field. As far as the line shape is concerned, this provides (in the large Doppler limit) a logarithmic "divergence," as noted in Ref. [2], with a width at half-maximum evolving as $\left(\gamma k u_{\mathrm{th}}\right)^{1 / 2}$. It also exhibits a close analogy with the well-known "logarithmic divergence" encountered in linear selective reflection spectroscopy [9]. Moreover, as in the FM selective reflection, the frequency derivation of the line shape associated with the experimental FM technique is particularly appropriate, as it turns the narrow sub-Doppler singularity into an effective Doppler-free structure [10].

To summarize, we have reported on an observation, in the optical domain, of the linear transient response of atoms in a room-temperature cell, a very general phenomenon in any cell of dilute vapor. This effect, which was only noticed in the microwave domain in the special case $L=\lambda / 2$, is actually observable in cells that are commonly produced, although they are very thin. Through the FM technique, the observation is proven to be feasible, even for relatively large values of $L / \lambda$ (notably for the large Doppler width to homogeneous width ratio). Hence, our experiments offer a method that combines Doppler-free spectroscopy in a vapor cell and linear response. In particular, and owing to the longer region of constructive interference interaction, the optical response should be larger than in the equivalent FM selective reflection spectroscopy. Such a method should be attractive to build up Doppler-free frequency standards that require ultralow incident power [11], or to be able to reach Dopplerfree molecular lines that are difficult to saturate.

Finally, we would like to point out that this linear transient regime exhibits marked differences with the previously analyzed incoherent optical pumping in similar thin cells. In particular, the observed sub-Doppler structure at line center results from the transient contribution of relatively fast atoms, as opposed, in the case of optical pumping, to a major contribution originating from an arbitrarily narrow velocity group $[5,12]$. In other words, the most unfrequent events, as in the desorption of an ultraslow atom, do not seem to play a special role in the present case, while in the case of an optical pumping mechanism, the statistical behavior in the thin cell [5] may be analyzed as a (truncated) Lévy flight [13].
[1] S. Briaudeau, D. Bloch, and M. Ducloy, Europhys. Lett. 35, 337 (1996).

[2] R. H. Romer and R. H. Dicke, Phys. Rev. 99, 532 (1955).

[3] For the 4-5 component, when hyperfine optical pumping can- not exist, the narrow resonance remains an increased absorption in a more extended intensity range, but the transition between the present linear behavior and the nonlinear behavior, due to Zeeman optical pumping, appears as a change in the line shape. 
[4] B. Zambon and G. Nienhuis, Opt. Commun. 143, 308 (1997); G. Nienhuis, in Quantum Optics of Confined Systems, Vol. 314 of NATO Advanced Study Institute, Series E: Applied Sciences, edited by M. Ducloy and D. Bloch (Kluwer, Dordrecht, 1996), pp. 341-353; see also T. Vartanyan and D. L. Lin, Phys. Rev. A 51, 1959 (1994).

[5] S. Briaudeau, Ph.D. dissertation, Université Paris-Nord, 1998 (unpublished); S. Briaudeau et al. (unpublished).

[6] The need to use a time-dependent theory in cw spectroscopy has been recognized in the context of optical pumping, by various groups; see, e.g., J. E. Bjorkholm, P. F. Liao, and A. Wojkaun, Phys. Rev. A 26, 2643 (1982).

[7] Note that the optimal case $L=\lambda / 2$ is the one considered in Ref. [2].

[8] In our case, the two cell windows are not perfectly parallel.
Taking into account an effective cell length increases the washing out of the $J_{3}$ contribution.

[9] M. F. H. Schuurmans, J. Phys. (Paris) 37, 469 (1975).

[10] Note that, in the principle, and due to the narrower structure of the $J_{3}$ term (see Fig. 2), $d J_{3} / d \delta$ may remain comparable with $d J_{2} / d \delta$, as long as $L / \lambda \leqslant k u_{\mathrm{th}} / \gamma$, but that any interferential averaging washes out the $J_{3}$ contribution.

[11] R. N. Li, S. T. Jia, D. Bloch, and M. Ducloy, Opt. Commun. 146, 186 (1998).

[12] S. Briaudeau, S. Saltiel, D. Bloch, and M. Ducloy, in Proceedings of XIII International Conference on Laser Spectroscopy, edited by Y. Z. Wang et al. (World Scientific, Singapore, in press).

[13] See, e.g., J-P. Bouchaud and A. Georges, Phys. Rep. 195, 127 (1990). 